\title{
Central Adiposity and Subclinical Cardiovascular Disease in Police Officers
}

\author{
Penelope Baughman, ${ }^{1,2}$ Desta Fekedulegn, ${ }^{2}$ Michael E. Andrew, ${ }^{2}$ Parveen Nedra Joseph, ${ }^{3}$ \\ Joan M. Dorn, ${ }^{4}$ John M. Violanti, ${ }^{4}$ and Cecil M. Burchfiel ${ }^{2}$ \\ ${ }^{1}$ Epidemic Intelligence Service, Centers for Disease Control and Prevention, Atlanta, GA 30333, USA \\ ${ }^{2}$ Biostatistics and Epidemiology Branch, Health Effects Laboratory Division, National Institute for Occupational Safety and Health, \\ Centers for Disease Control and Prevention, Morgantown, WV 26505, USA \\ ${ }^{3}$ The University of Chicago Comprehensive Cancer Center, The University of Chicago, Chicago, IL 60637, USA \\ ${ }^{4}$ Department of Social and Preventive Medicine, School of Public Health and Health Professions, The State University of \\ New York at Buffalo, Buffalo, NY 14214, USA
}

Correspondence should be addressed to Penelope Baughman; pbaughman@cdc.gov

Received 4 December 2012; Accepted 28 December 2012

Academic Editors: N. Aires and S. Straube

Copyright (C) 2013 Penelope Baughman et al. This is an open access article distributed under the Creative Commons Attribution License, which permits unrestricted use, distribution, and reproduction in any medium, provided the original work is properly cited.

Given the associations between obesity and cardiovascular disease (CVD), we evaluated a related but less well-established association between waist circumference and brachial artery reactivity (BAR), a functional measurement of subclinical CVD, where lower levels indicate dysfunction. Regression models examined trends in mean BAR across waist circumference tertiles in police officers, a high-stress occupational group with increased risk for CVD. Mean BAR decreased across increasing waist tertiles among men, but not women, and this association was stronger among officers who consumed more alcohol. Larger waist circumference may be associated with lower BAR, providing an opportunity for intervention prior to disease development.

\section{Objective}

Policing in the United States presently consists of over 794,000 sworn officers and is projected to rise to 853,000 officers by 2020 [1]. Police officers have high rates of cardiovascular disease (CVD), yet reasons for this finding are not fully understood. Evidence suggests that overweight and obesity may be even more common among police officers than in the general population [2]. As this excess may partly explain the high rates of CVD, we evaluated the association between waist circumference, an indicator of central adiposity, and brachial artery reactivity (BAR), a subclinical marker of CVD. BAR is a measure of the dilation of the brachial artery after occlusion using a blood pressure cuff. Lower BAR indicates dysfunction and early-stage atherosclerosis [3]. Knowledge of associations between obesity and CVD [47] leads us to hypothesize that larger waist circumference may be associated with lower BAR.

\section{Methods}

Our study combined data from two cross-sectional studies of police officers in a midsized urban department. The first study, in 1999-2000, included a random sample of 115 officers from 934 employed officers, with oversampling for women. Participation was $100 \%$. The second study, in 2001-2003, included 100 officers, 87 of whom were examined previously. Participants were sworn officers and willing to participate. The studies were approved by The State University of New York at Buffalo Institutional Review Board and the National Institute for Occupational Safety and Health Human Subjects Review Board [8]. Our sample included 70 officers, after excluding 17 officers with missing data for waist circumference or BAR. Data were from 1999-2000, except for BAR which was measured during the second study.

Waist circumference was the mean of two measurements, in centimeters $(\mathrm{cm})$, taken while standing and after exhalation. Body mass index (BMI) and abdominal height were 
TABLE 1: Demographic, lifestyle, occupational, and physical characteristics of participating police officers.

\begin{tabular}{|c|c|c|c|c|}
\hline Characteristic & $\begin{array}{c}\text { Men } \\
(n=40)\end{array}$ & $\begin{array}{l}\text { Women } \\
(n=30)\end{array}$ & $\begin{array}{c}\text { All } \\
(n=70)\end{array}$ & $P$ value ${ }^{\mathrm{a}}$ \\
\hline Age, mean (SD), $y$ & $43.88(9.16)$ & $43.90(5.99)$ & $43.89(7.91)$ & 0.99 \\
\hline \multicolumn{5}{|l|}{ Race/ethnicity } \\
\hline White & $34(85.0)$ & $22(73.3)$ & $56(80.0)$ & \multirow{3}{*}{0.04} \\
\hline Black & $3(7.5)$ & $8(26.7)$ & $11(15.7)$ & \\
\hline Hispanic & $3(7.5)$ & $0(0.0)$ & $3(4.3)$ & \\
\hline \multicolumn{5}{|l|}{ Education } \\
\hline$\leq$ High school/GED & $6(15.0)$ & $6(20.0)$ & $12(17.1)$ & \multirow{3}{*}{0.50} \\
\hline College, $<4 \mathrm{y}$ & $11(27.5)$ & $11(36.7)$ & $22(31.4)$ & \\
\hline College, $\geq 4 \mathrm{y}$ & $23(57.5)$ & $13(43.3)$ & $36(51.4)$ & \\
\hline \multicolumn{5}{|l|}{ Smoking } \\
\hline Ever & $17(42.5)$ & $19(63.3)$ & $36(51.4)$ & \multirow{2}{*}{0.08} \\
\hline Never & $23(57.5)$ & $11(36.7)$ & $34(48.6)$ & \\
\hline Physical activity ${ }^{\mathrm{b}}$, mean (SD), h/wk & $7.11(10.77)$ & $6.76(9.01)$ & $6.96(9.98)$ & 0.88 \\
\hline \multicolumn{5}{|l|}{ Physical activity index, $(\mathrm{h} / \mathrm{wk}) \times$ intensity $^{\mathrm{c}}$} \\
\hline $0-1.50$ & $9(22.5)$ & $9(30.0)$ & $18(25.7)$ & \multirow{4}{*}{0.12} \\
\hline $1.51-6.50$ & $13(32.5)$ & $4(13.3)$ & $17(24.3)$ & \\
\hline $6.51-14.50$ & $7(17.5)$ & $11(36.7)$ & $18(25.7)$ & \\
\hline$\geq 14.51$ & $11(27.5)$ & $6(20.0)$ & $17(24.3)$ & \\
\hline \multicolumn{5}{|l|}{ Alcohol intake, drinks/wk } \\
\hline All participants, mean (SD) & $3.45(4.68)$ & $2.04(3.31)$ & $2.84(4.18)$ & 0.16 \\
\hline 0 & $7(17.5)$ & $11(36.7)$ & $18(25.7)$ & \multirow{4}{*}{0.23} \\
\hline$<1$ & $11(27.5)$ & $8(26.7)$ & $19(27.1)$ & \\
\hline $1-5$ & $15(37.5)$ & $9(30.0)$ & $24(34.3)$ & \\
\hline$\geq 6$ & $7(17.5)$ & $2(6.7)$ & $9(12.9)$ & \\
\hline Traumatic police incidents, mean (SD), events/y & $4.68(2.19)$ & $3.30(2.15)$ & $4.09(2.26)$ & 0.01 \\
\hline \multicolumn{5}{|l|}{ Waist circumference, $\mathrm{cm}$} \\
\hline All participants, mean (SD) & $95.06(10.04)$ & $81.95(10.77)$ & $89.44(12.18)$ & $<0.001$ \\
\hline Normal ( $<102$ in men, $<88$ in women) & $29(72.5)$ & $22(73.3)$ & $51(72.9)$ & \multirow{2}{*}{0.94} \\
\hline Abdominal obesity ( $\geq 102$ in men, $\geq 88$ in women) & $11(27.5)$ & $8(26.7)$ & $19(27.1)$ & \\
\hline Brachial artery reactivity, mean (SD), \% increase & $4.57(3.47)$ & $4.16(3.53)$ & $4.39(3.48)$ & 0.63 \\
\hline
\end{tabular}

SD: standard deviation; $\mathrm{cm}$ : centimeters.

${ }^{\mathrm{a}}$ Test compares gender-specific values. $P$ values from chi-squared test or Fisher's exact test for categorical variables and from $t$-test for continuous variables.

${ }^{\mathrm{b}}$ Moderate intensity or greater.

${ }^{\mathrm{c}}$ Intensity categorized as moderate, hard, and very hard.

also measured but were not selected because BMI does not distinguish between fat- and lean-mass [9] and because standardized criteria for abdominal obesity $(\geq 102 \mathrm{~cm}$ in men, $\geq 88 \mathrm{~cm}$ in women) are more clearly defined for waist circumference [10]. BAR was measured with flow-mediated dilation (FMD), a functional measure of subclinical CVD involving compression of the brachial artery. Arterial diameter was monitored continuously using standardized [11], noninvasive ultrasound scans during the eight-minute procedure: one-minute baseline, four-minute occlusion with a blood pressure cuff, and three-minute period after cuff deflation. BAR was evaluated as percent change in diameter from baseline to maximum diameter following cuff deflation.

Analyses of variance and covariance were used to estimate and describe mean BAR across tertiles of waist circumference. Linear regression assessed the trend, $P<0.05$. Models were sex stratified and adjusted for age, smoking status (ever/never), and physical activity (composite of hours per week and intensity) which were obtained by selfadministered questionnaire. Based on biological plausibility, tests for interaction were conducted for alcohol intake (drinks per week), smoking status, and occupational stress (traumatic police incidents in past year), $P \leq 0.20$. Due to limited sample size, effect modification was evaluated among men and women combined. Analyses were conducted using SAS, 9.3 (SAS Inc., Cary, NC, USA).

\section{Results}

Mean age for the 70 officers in our study was 43.9 years, and $57.1 \%$ were men (Table 1). A greater percentage of women reported current or former smoking. Reported average alcohol intake was less than 3.5 drinks per week. Approximately, $27 \%$ of the officers had abdominal obesity (waist 
TABLE 2: Mean percent change in brachial artery reactivity (BAR) by tertiles of waist circumference in police officers.

\begin{tabular}{|c|c|c|c|c|c|}
\hline \multirow{2}{*}{ Waist tertile $^{\mathrm{a}}$} & \multirow{2}{*}{$N$} & \multicolumn{2}{|c|}{ Unadjusted } & \multicolumn{2}{|c|}{ Multivariate adjusted $^{\mathrm{b}}$} \\
\hline & & Mean (SD) & $P$ value $^{\mathrm{c}}$ & Mean (SE) & $P$ value ${ }^{c}$ \\
\hline \multicolumn{6}{|l|}{ Men } \\
\hline Low & 14 & $5.61(4.47)$ & \multirow{3}{*}{0.04} & $5.96(0.94)$ & \multirow{3}{*}{0.06} \\
\hline Medium & 13 & $4.69(2.97)$ & & $4.26(0.95)$ & \\
\hline High & 13 & $3.32(2.42)$ & & $3.37(0.94)$ & \\
\hline \multicolumn{6}{|l|}{ Women } \\
\hline Low & 10 & $4.99(2.95)$ & \multirow{3}{*}{0.83} & $4.99(1.16)$ & \multirow{3}{*}{0.87} \\
\hline Medium & 10 & $3.32(4.78)$ & & $3.47(1.16)$ & \\
\hline High & 10 & $4.18(2.67)$ & & $4.02(1.16)$ & \\
\hline \multicolumn{6}{|c|}{ Waist tertile by alcohol intake $\leq$ median $^{\mathrm{d}}$} \\
\hline Low & 12 & $3.55(3.23)$ & \multirow{3}{*}{0.74} & $3.31(1.16)$ & \multirow{3}{*}{0.27} \\
\hline Medium & 13 & $4.97(5.46)$ & & $4.40(1.14)$ & \\
\hline High & 12 & $5.11(2.57)$ & & $5.96(1.19)$ & \\
\hline \multicolumn{6}{|c|}{ Waist tertile by alcohol intake $>$ median } \\
\hline Low & 12 & $5.57(2.75)$ & \multirow{3}{*}{0.01} & $5.56(0.78)$ & \multirow{3}{*}{0.01} \\
\hline Medium & 9 & $4.82(3.32)$ & & $5.20(0.95)$ & \\
\hline High & 12 & $2.40(1.73)$ & & $2.12(0.81)$ & \\
\hline
\end{tabular}

SD: standard deviation; SE: standard error.

${ }^{\mathrm{a}}$ Waist tertiles were $80-89.4,89.5-97.9$, and $98-126 \mathrm{~cm}$ for men, $64.5-74.9,75-86.9$, and $87-110.1 \mathrm{~cm}$ for women, and $64.5-85.1,85.2-92.4$, and $92.5-126 \mathrm{~cm}$ for all police officers.

${ }^{\mathrm{b}}$ Adjusted for age, smoking, and physical activity.

${ }^{\mathrm{c}} P$ values are from linear regression models.

${ }^{\mathrm{d}}$ Median value was 0.88 drinks per week. $P$ value for interaction with alcohol was 0.30 .

circumference $\geq 102 \mathrm{~cm}$ in men and $\geq 88 \mathrm{~cm}$ in women). The mean percent change in BAR was similar for men (4.57\%) and women (4.16\%). Among several demographic, lifestyle, and CVD risk factors, the only statistically significant difference between study participants $(n=70)$ and nonparticipants $(n=35)$ was in women where participants were older on average.

Adjusted mean percent change in BAR decreased (5.96\%, $4.26 \%$, and $3.37 \%$; $P$ trend $=0.06$ ) across increasing waist tertiles (80-89.4, 89.5-97.9, and 98-126 cm) among men (Table 2). The trend was not statistically significant in women. Effect modification was then assessed among all officers in models stratified on median alcohol intake (test for interaction: $P=0.30$ ). Officers who had intake above the median (0.88 drinks per week) had a significant decline in adjusted mean BAR (5.56\%, 5.20\%, and 2.12\%; $P=0.01$ ) across increasing waist tertiles, whereas officers with lower intake had a nonsignificant increase. Tests for statistical interaction involving smoking and traumatic police incidents were not statistically significant.

\section{Discussion}

Although previous studies demonstrate associations between obesity and CVD $[4,5]$, an association between waist circumference and BAR was observed only in men in our study, even though similar percentages of men and women had an elevated waist circumference. Given that these women were largely of premenopausal age, estrogen could have been an effect modifier [12]. However, the statistical interaction with sex was not statistically significant $(P=0.22)$. The results for alcohol intake were interesting because reported intake was fairly low. Yet, the lowest adjusted mean percent change in BAR was in officers with higher intake and high waist circumference, and, though nonsignificant, the largest change was in officers with lower intake and high waist circumference. Despite assurances of confidentiality, alcohol intake may have been underreported. Although occupational stress, represented as traumatic police incidents, was not an effect modifier in our study, it may be in the causal chain as it may lead to greater central deposition of adipose tissue with increased cortisol levels [13].

Although few studies have focused on this association, our results are similar to those from a large community-based study that also identified an inverse association between waist circumference and BAR, but the association was present in men and women unlike our results which are based on a smaller sample size [6]. In a study of healthy male firefighters, indexes of adiposity were associated with vascular dysfunction, but not specifically with BAR [7]. Neither of these studies specifically examined the effect of alcohol intake on the association between waist circumference and BAR. A larger, more detailed investigation may be beneficial in future studies, particularly with respect to intake patterns including both frequency and intensity. Together, these characteristics have been associated with the distribution of body fat [14]. 
Strengths of our study include standardized measurement of waist circumference and BAR and oversampling for women. However, lifestyle factors were self-reported and could have changed somewhat over the two- to three-year period. The relatively small sample size and cross-sectional design are potential limitations. Use of data from the two study periods could have resulted in associations that were underestimated, but the average percent change in individual body weight between the studies was rather small at $2.3 \%$.

\section{Conclusion}

Results indicate that in this sample of police officers, larger waist circumference was associated with lower BAR in men, and this association was stronger for officers who consumed more alcohol, a CVD risk factor. The FMD procedure is a research tool that can be useful in characterizing relationships between CVD risk factors, such as central adiposity, and subclinical disease in populations with increased CVD risk [15]. Future studies investigating the relationship between waist circumference and BAR would benefit from a larger sample size and prospective design. Such studies may provide evidence that could be useful in planning intervention studies to determine whether public health efforts to reduce waist circumference may lead to improvements in BAR and thus lower CVD risk.

\section{Disclaimer}

The findings and conclusions in this paper are those of the authors and do not necessarily represent the views of the National Institute for Occupational Safety and Health.

\section{Acknowledgment}

This work was supported by the National Institute for Occupational Safety and Health (NIOSH), Grant no. 1R030H003772-01 and Contract no. HELD01B0088.

\section{References}

[1] U.S. Bureau of Labor Statistics, "Occupational outlook handbook, police and detectives," 2010-2011, http://www.bls.gov/ ooh/protective-service/police-and-detectives.htm.

[2] F. H. Zimmerman, "Cardiovascular disease and risk factors in law enforcement personnel: a comprehensive review," Cardiology in Review, vol. 20, no. 4, pp. 159-166, 2012.

[3] R. A. Vogel, "Measurement of endothelial function by brachial artery flow-mediated vasodilation," The American Journal of Cardiology, vol. 88, no. 2, pp. 31E-34E, 2001.

[4] P. W. Wilson, R. B. D'Agostino, L. Sullivan, H. Parise, and W. B. Kannel, "Overweight and obesity as determinants of cardiovascular risk: the Framingham experience," Archives of Internal Medicine, vol. 162, no. 16, pp. 1867-1872, 2002.

[5] C. E. Lewis, K. M. McTigue, L. E. Burke et al., "Mortality, health outcomes, and body mass index in the overweight range: a science advisory from the American Heart Association," Circulation, vol. 119, no. 25, pp. 3263-3271, 2009.
[6] N. I. Parikh, M. J. Keyes, M. G. Larson et al., "Visceral and subcutaneous adiposity and brachial artery vasodilator function," Obesity, vol. 17, no. 11, pp. 2054-2059, 2009.

[7] B. J. Martin, S. Verma, F. Charbonneau, L. M. Title, E. M. Lonn, and T. J. Anderson, "The relationship between anthropometric indexes of adiposity and vascular function in the FATE cohort," Obesity. In press.

[8] J. M. Violanti, C. M. Burchfiel, D. B. Miller et al., "The Buffalo Cardio-Metabolic Occupational Police Stress (BCOPS) pilot study: methods and participant characteristics," Annals of Epidemiology, vol. 16, no. 2, pp. 148-156, 2006.

[9] S. B. van Dijk, T. Takken, E. C. Prinsen, and H. Wittink, "Different anthropometric adiposity measures and their association with cardiovascular disease risk factors: a meta-analysis," Netherlands Heart Journal, vol. 20, no. 5, pp. 208-218, 2012.

[10] S. M. Grundy, J. I. Cleeman, S. R. Daniels et al., "Diagnosis and management of the metabolic syndrome: An American Heart Association/National Heart, Lung, and Blood Institute scientific statement," Circulation, vol. 112, no. 17, pp. 2735-2752, 2005.

[11] P. N. Joseph, J. Dorn, J. M. Violanti, M. E. Andrew, and W. A. Riley, "Examining the brachial artery reactivity curve measured by b-mode ultrasound in the Buffalo Cardio-Metabolic Occupational Police Stress (BCOPS) pilot study," Journal for Vascular Ultrasound, vol. 29, no. 4, pp. 199-206, 2005.

[12] D. Xing, S. Nozell, Y. F. Chen, F. Hage, and S. Oparil, "Estrogen and mechanisms of vascular protection," Arteriosclerosis, Thrombosis, and Vascular Biology, vol. 29, no. 3, pp. 289-295, 2009.

[13] G. P. Chrousos and P. W. Gold, "A healthy body in a healthy mind-and vice versa - the damaging power of 'uncontrollable' stress," Journal of Clinical Endocrinology and Metabolism, vol. 83, no. 6, pp. 1842-1845, 1998.

[14] J. M. Dorn, K. Hovey, P. Muti et al., "Alcohol drinking patterns differentially affect central adiposity as measured by abdominal height in women and men," The Journal of Nutrition, vol. 133, no. 8, pp. 2655-2662, 2003.

[15] D. Tousoulis, C. Antoniades, C. Vlachopoulos, and C. Stefanadis, "Flow mediated dilation and carotid intima media thickness:clinical markers or just research tools?" International Journal of Cardiology. In press. 


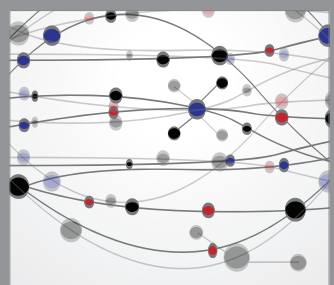

The Scientific World Journal
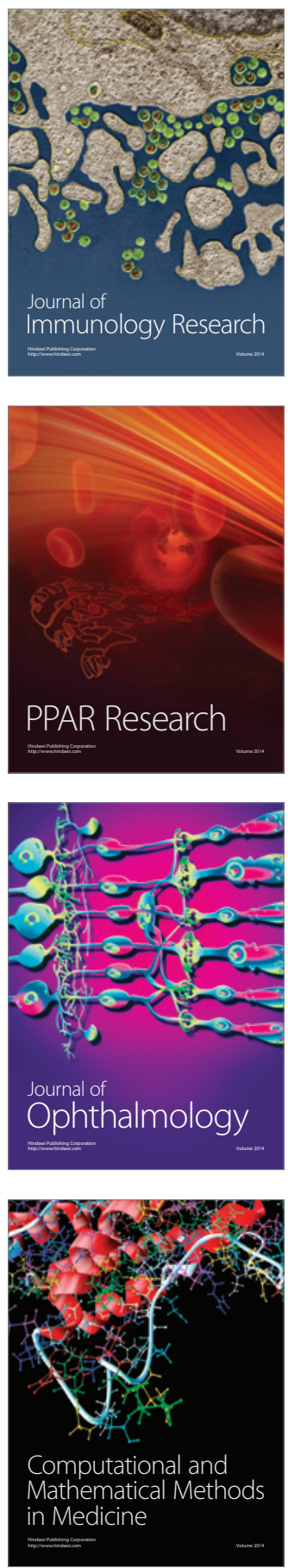

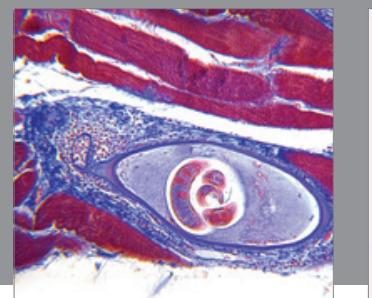

Gastroenterology

Research and Practice
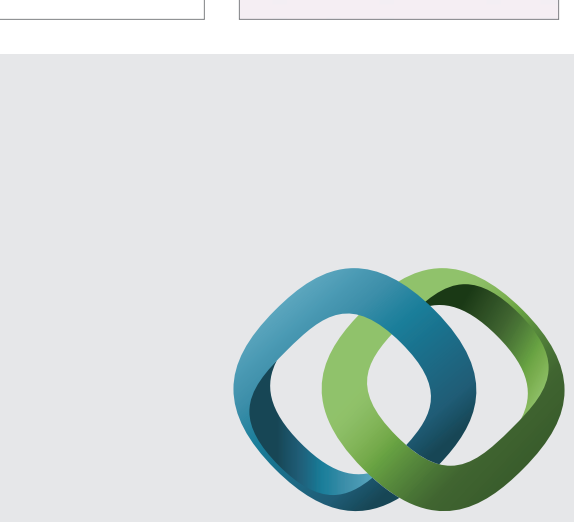

\section{Hindawi}

Submit your manuscripts at

http://www.hindawi.com
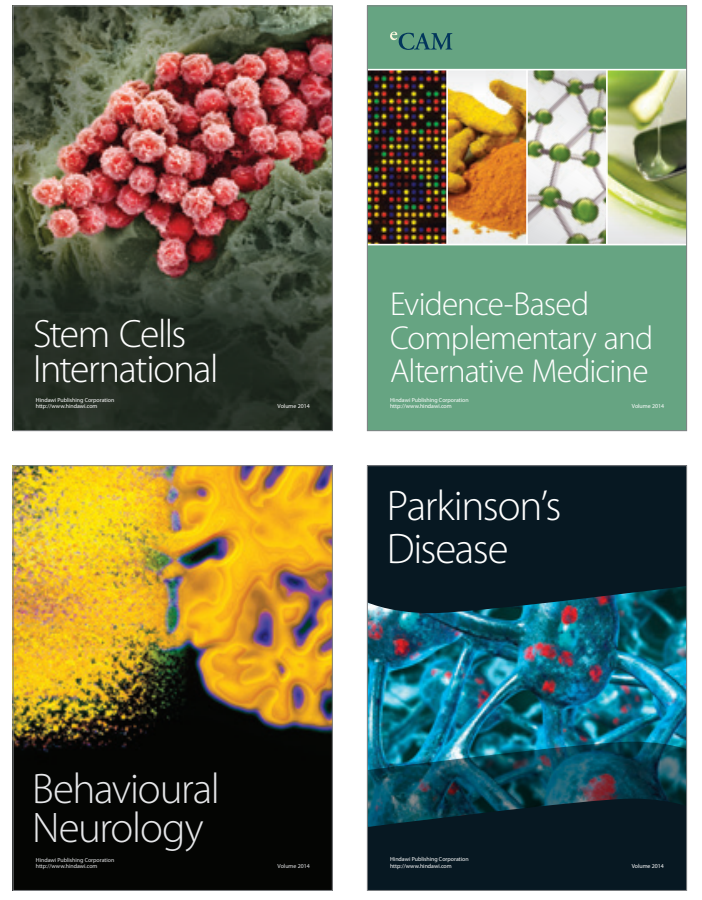
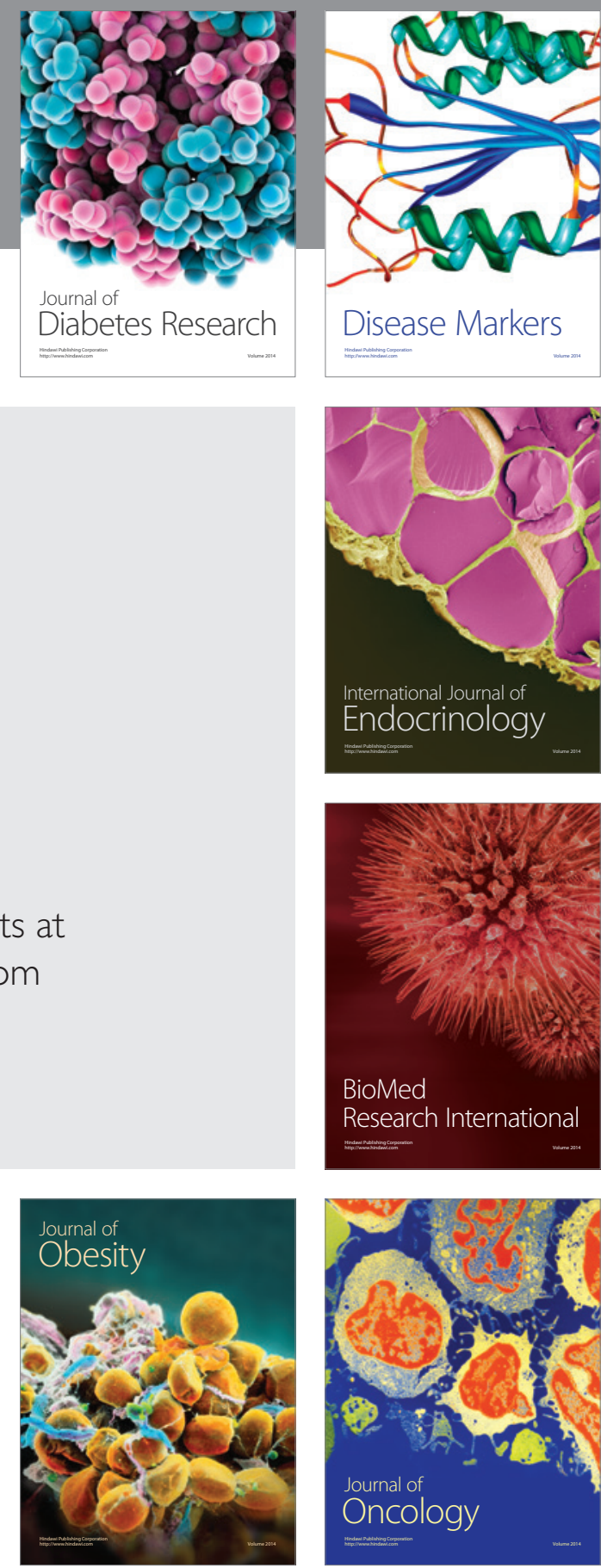

Disease Markers
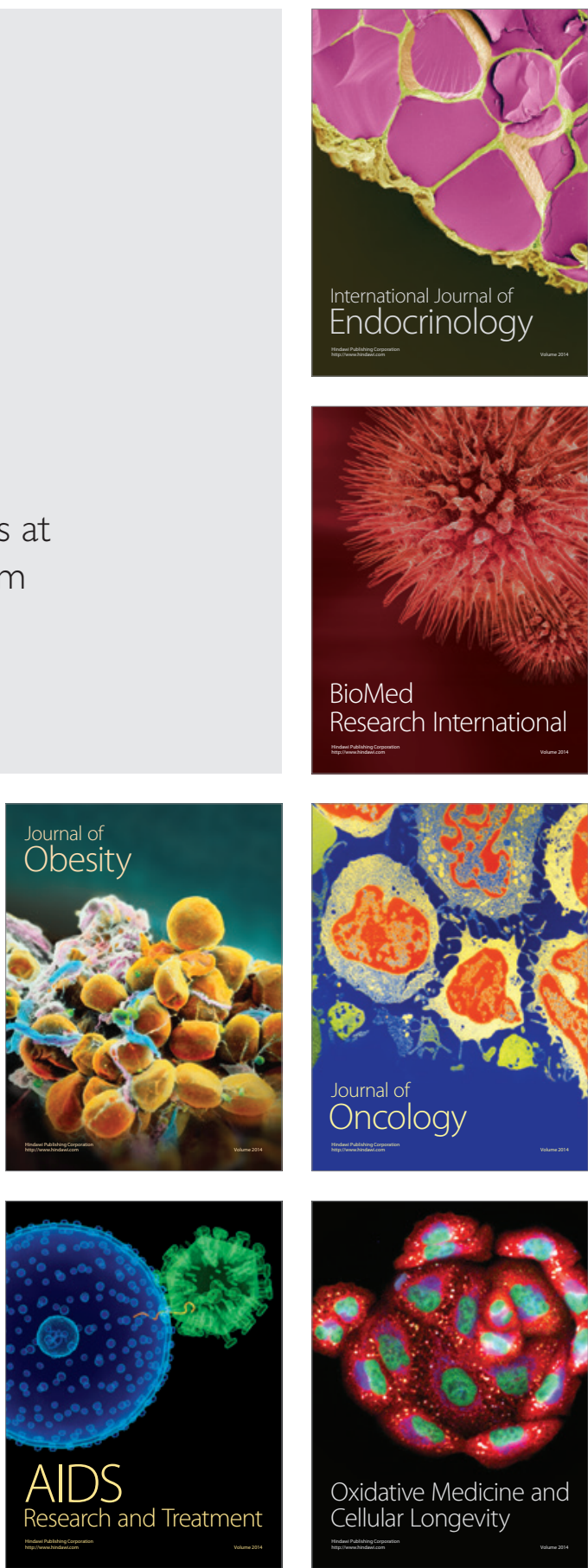